\title{
On the Propagation of Round-Off Errors in the Numerical Treatment of the Wave Equation
}

\author{
By Arnold N. Lowan
}

Abstract. An upper bound of the norm of the error vector after $n$ time steps is $\frac{1}{2}(n+1)(n+2)\left\|\boldsymbol{\delta}^{*}\right\|$. For the explicit scheme $\delta^{*}=\left\|\boldsymbol{\delta}^{*}\right\|=3 \times \frac{1}{2} \times 10^{-p}$ where $p$ is the number of decimals carried in the computations. For the implicit scheme $\delta^{*}=\left\|\boldsymbol{\delta}^{*}\right\|$ is an upper bound of the errors which arise both from using approximations to $A^{-1}$ and $A^{-1} B$ in the determination of $\mathbf{u}_{k+1}$ from equation $\left(6^{*}\right)$ and from rounding off the values of the products and quotients involved in the computation of the components of $\mathbf{u}_{k+1}$.

Consider the numerical treatment of the differential equation of wave motion

$$
\frac{\partial^{2} u}{\partial t^{2}}=c^{2} \frac{\partial^{2} u}{\partial x^{2}}
$$$$
0 \leqq x \leqq a, \quad t>0
$$

the solution of which is required to satisfy the following initial and boundary conditions

$$
\begin{aligned}
u(x, 0) & =f(x) \\
u_{t}(x, 0) & =g(x) \\
u(0, t) & =u(a, t)=0 .
\end{aligned}
$$

With the differential equation (1) we will associate either of the following two difference analogs [1]

$$
\begin{gathered}
u_{h, k+1}-2 u_{h, k}+u_{h, k-1}=R^{2}\left(u_{h-1, k}-2 u_{h, k}+u_{h+1, k}\right) \\
u_{h, k+1}-2 u_{h, k}+u_{h, k-1}=\frac{R^{2}}{2}\left(u_{h-1, k+1}-2 u_{h, k+1}+u_{h+1, k+1}\right. \\
\left.+u_{h-1, k-1}-2 u_{h, k-1}+u_{h+1, k-1}\right)
\end{gathered}
$$

where $R=c \Delta t / \Delta x$ and $u_{h, k}=u(h \Delta x, k \Delta t)$ with $(M+1) \Delta x=a$.

The difference counterpart of (3) will be taken in the form

$$
\frac{u_{h, 1}-u_{h, 0}}{\Delta t}=g(h \Delta x) \text {; }
$$

whence

$$
u_{h, 1}=u_{h, 0}+g(h \Delta x) \Delta t=f(h \Delta x)+g(h \Delta x) \Delta t .
$$

The difference equations (5) and (6) may be written in the compact forms

$$
\begin{aligned}
\mathbf{u}_{k+1} & =A \mathbf{u}_{k}-\mathbf{u}_{k-1} \\
A \mathbf{u}_{k+1} & =4 \mathbf{u}_{k}+B \mathbf{u}_{k-1} .
\end{aligned}
$$

Received August 17, 1959; in revised form, December 24, 1959. 
In $\left(5^{*}\right) A$ is a tridiagonal matrix whose elements on the principal diagonal are $=2\left(1-R^{2}\right)$ and whose elements off the principal diagonal are $=R^{2}$ and $\mathfrak{u}_{k}$ is the vector whose components are the values of $u(x, t)$ at time $t=k \Delta t$ at the lattice points $x=h \Delta x, h=1,2,3 \cdots$. In $\left(6^{*}\right) A$ is a tridiagonal matrix whose elements on the principal diagonal are $=2\left(1+R^{2}\right)$ while the elements off the principal diagonal are $=-R^{2}$ and $B$ is a tridiagonal matrix whose elements on the principal diagonal are $=-2\left(1+R^{2}\right)$ while the elements off the principal diagonal are $=R^{2}$.

Consider first the explicit difference scheme $\left(5^{*}\right)$. Since both $\mathbf{u}_{0}$ and $\mathbf{u}_{1}$ are known, $\left(5^{*}\right)$ will yield in succession $\mathbf{u}_{2}, \mathbf{u}_{3} \cdots$. Specifically,

$$
\left\{\begin{array}{l}
\mathbf{u}_{2}=A \mathbf{u}_{1}-\mathbf{u}_{0} \\
\mathbf{u}_{3}=A \mathbf{u}_{2}-\mathbf{u}_{1} \\
\vdots \\
\mathbf{u}_{n}=A \mathbf{u}_{n-1}-\mathbf{u}_{n-2}
\end{array}\right.
$$

It is reasonable to assume that the components of $\mathbf{u}_{0}$ are exact while those of $\mathbf{u}_{1}$, obtained from (7), have been rounded off to the number of decimal places in be carried in the computations. Let $\mathbf{u}_{1}{ }^{*}$ denote the vector whose components are the rounded off values of the components of $\mathbf{u}_{1}$. It is then easily seen that we introduce two types of errors in the evaluation of $\mathbf{u}_{2}$. A first error is due to using $\mathbf{u}_{1} *$ in lieu of $\mathfrak{u}_{1}$. A second error is introduced as a result of rounding off of the values of the products involved in the expression of $u_{h, k+1}$ obtained from (5) to the number of decimal places carried in the computations. Thus, in lieu of the exact vector $\mathbf{u}_{2}$, the first step in the sequence of operations (8) yields the vector $\mathbf{u}_{2}{ }^{*}=A \mathbf{u}_{1}{ }^{*}$ $\mathbf{u}_{0}+\boldsymbol{\delta}_{2}$ where $\boldsymbol{\delta}_{2}$ is the error vector whose components are the round-off errors just discussed. Similarly, error vectors are introduced in each of the successive steps in the sequence of operations (8). Thus

$$
\left\{\begin{array}{l}
\mathbf{u}_{2}{ }^{*}=A \mathbf{u}_{1}{ }^{*}-\mathbf{u}_{0}+\boldsymbol{\delta}_{2} \\
\mathbf{u}_{3}{ }^{*}=A \mathbf{u}_{2}{ }^{*}-\mathbf{u}_{1}{ }^{*}+\boldsymbol{\delta}_{3} \\
\vdots \\
\mathbf{u}_{n}{ }^{*}=A \mathbf{u}_{n-1}^{*}-\mathbf{u}_{n-2}^{*}+\boldsymbol{\delta}_{n}
\end{array}\right.
$$

If we put

$$
\mathbf{E}_{n}=\mathbf{u}_{n}^{*}-\mathbf{u}_{n}
$$

then from (8) and (9) it follows that

$$
\mathrm{E}_{n}=A \mathrm{E}_{n-1}-\mathbf{E}_{n-2}+\mathbf{\delta}_{n}
$$

In entirely similar manner it may be shown that the counterpart of (11) for the implicit scheme $\left(6^{*}\right)$ is

$$
\mathbf{E}_{n}=4 A^{-1} \mathbf{E}_{n-1}+A^{-1} B \mathbf{E}_{n-2}+\boldsymbol{\delta}_{n} .
$$

There is, however an important distinction between (11) and (12); whereas in (11) the components of $\boldsymbol{\delta}_{n}$ are round-off errors as above explained, in (12) the components of $\boldsymbol{\delta}_{n}$ are the aggregate of the errors arising both from using approximations to $A^{-1}$ and $A^{-1} B$ in the determination of $\mathbf{u}_{k+1}$ and the round-off errors 
introduced as a result of rounding-off the values of the products and quotients involved in the computation of the components of $\mathbf{u}_{k+1}$.

The error equations (11) and (12) are of the form

$$
\mathbf{E}_{n}=M \mathrm{E}_{n-1}+N \mathrm{E}_{n-2}+\boldsymbol{\delta}_{n} \text {. }
$$

If in (13) we put in succession $n=2,3,4, \cdots$ and write $\delta_{1}$ for $E_{1}$, it may be shown by induction that

$$
\mathbf{E}_{n}=P_{n-1}(M, N) \boldsymbol{\delta}_{1}+P_{n-2}(M, N) \boldsymbol{\delta}_{2}+\cdots \boldsymbol{\delta}_{n}
$$

or

$$
\mathbf{E}_{n}=\sum_{p=0}^{n} P_{p}(M, N) \boldsymbol{\delta}_{n-p}
$$

where

$$
\begin{aligned}
P_{n}(M, N)=M^{n}+C_{n-1}^{1} & M^{n-2} N \\
& +C_{n-2}^{2} M^{n-4} N^{2}+\cdots C_{n-8}^{8} M^{n-8} N^{8}+\cdots
\end{aligned}
$$

or

$$
P_{n}(M, N)=\sum_{s=0}^{(n / 2)} C_{n-8}^{s} M^{n-2 s} N^{s}
$$

where $(n / 2)$ denotes the largest integer in $n / 2$, where $C_{n}{ }^{0}=1$ and $C_{m}{ }^{n}$ denote the binomial coefficient $m(m-1)(m-2) \cdots(m-n+1) / n$ !.

We shall prove that if $M$ and $N$ have the same eigenvectors, then

$$
\left\|P_{p}(M, N) \boldsymbol{\delta}_{n-p}\right\| \leqq\left\|\boldsymbol{\delta}_{n-p}\right\| \cdot(p+1)
$$

where for any $M$-dimensional vector $\phi$, its norm $\|\boldsymbol{\phi}\|$ is defined by

$$
\|\phi\|=\sqrt{(\phi, \phi)}=\sqrt{\frac{1}{M} \sum_{h=1}^{M}\left(\phi_{h}\right)^{2}}
$$

the $\phi_{h}$ 's being the components of $\phi$, provided that the roots of the quadratic equation

$$
x^{2}-\lambda_{r} x-\mu_{r}=0
$$

where the $\lambda_{r}$ 's and $\mu_{r}$ 's, the eigenvalues of $M$ and $N$ respectively, are either numerically equal to or smaller than unity (if real) or have a modulus equal to or smaller than unity (if complex). Indeed, let

$$
\boldsymbol{\delta}_{n-p}=\sum_{r=1}^{M} \alpha_{r}^{(n-p)} \mathrm{w}_{r}
$$

where the $\mathrm{w}_{r}$ 's are the normalized eigenvectors of the matrices $M$ and $N$. From (19) and $\left(14^{*}\right)$ we get

$$
P_{p}(M, N) \boldsymbol{\delta}_{n-p}=\sum_{s=0}^{(p / 2)} \sum_{r=1}^{M} C_{p-s}^{s} \alpha_{r}^{(n-p)} M^{p-2 s} N^{s} \mathrm{w}_{r}
$$


But

$$
M^{p-28} N^{s} \mathrm{~W}_{r}=M^{p-2 s} \mu_{r}^{8} \mathrm{~W}_{r}=\lambda_{r}^{p-2 s} \mu_{r}^{s} \mathrm{~W}_{r}
$$

whence

$$
\begin{aligned}
P_{p}(M, N) \boldsymbol{\delta}_{n-p} & =\sum_{r=1}^{M} \alpha^{(n-p)} \mathrm{w}_{r} \sum_{s=0}^{(p / 2)} C_{p-s}^{s} \lambda_{r}^{p-2 s} \mu_{r}^{8} \\
& =\sum_{r=1}^{M} \beta_{r}(p) \alpha_{r}^{(n-p)} \mathrm{w}_{r}
\end{aligned}
$$

It may be proved by induction that

$$
\beta_{r}(p)=\sum_{s=0}^{(p / 2)} C_{p-s}^{s} \lambda_{r}^{p-2 s} \mu_{r}^{8}=\frac{x_{1, r}^{p+1}-x_{2, r}^{p+1}}{x_{1, r}-x_{2, r}}=\sum_{\sigma=0}^{p} x_{1, r}^{\sigma} x_{2, r}^{p-\sigma}
$$

where $x_{1, r}$ and $x_{2, r}$ are the roots of the quadratic equation (18). From (22) it is clear that if these roots are numerically smaller than unity then

$$
\left|\beta_{r}(p)\right|<p+1 \text {; }
$$

and furthermore $\beta_{r}(p) \rightarrow 0$ as $p \rightarrow \infty$. In view of (23), (21) yields

$$
\left\|P_{p}(M, N) \boldsymbol{\delta}_{n-p}\right\|=\sqrt{\sum_{r=1}^{M}\left[\beta_{r}(p)\right]^{2}\left[\alpha_{r}^{(n-p)}\right]^{2}} \leqq(p+1) \sqrt{\sum_{r=1}^{M}\left[\alpha_{r}^{(n-p)}\right]^{2}} ;
$$

or

$$
\left\|P_{p}(M, N) \boldsymbol{\delta}_{n-p}\right\| \leqq(p+1)\left\|\boldsymbol{\delta}_{n-p}\right\| .
$$

From $\left(14^{*}\right)$ the Minkowski inequality yields

$$
\left\|\mathbf{E}_{n}\right\| \leqq \sum_{p=0}^{n}\left\|P_{p}(M, N) \boldsymbol{\delta}_{n-p}\right\|
$$

whence, in view of (16)

$$
\left\|\mathbf{E}_{n}\right\| \leqq \sum_{p=0}^{n}(p+1)\left\|\boldsymbol{\delta}_{n-p}\right\|
$$

and a fortiori

$$
\left\|\mathbf{E}_{n}\right\| \leqq\left\|\boldsymbol{\delta}^{*}\right\| \sum_{p=0}^{n}(p+1)=\frac{(n+1)(n+2)}{2}\left\|\boldsymbol{\delta}^{*}\right\|,
$$

where $\left\|\delta^{*}\right\|$ is the largest of the sequence $\left\|\delta_{1}\right\|,\left\|\delta_{2}\right\| \cdots\left\|\delta_{n}\right\|$. If $\delta^{*}$ denotes an upper bound of the components of all the vectors $\delta_{p}$, it is readily seen that

$$
\left\|\boldsymbol{\delta}^{*}\right\| \leqq \delta^{*}
$$

Furthermore, since

$$
\left\|\mathbf{E}_{n}\right\|=\left\{\frac{1}{M} \sum_{h=1}^{M}\left(E_{n h}\right)^{2}\right\}^{1 / 2}
$$

where the $E_{n h}$ 's are the components of $E_{n}$, it is clear that the maximum of any of the components is obtained by assuming that all but one of the components are $=0$. 
Calling the maximum value of the components $E_{n}{ }^{*}$ we finally get

$$
E_{n}{ }^{*} \leqq \frac{1}{2}(n+1)(n+2) \sqrt{M} \delta^{*}
$$

The second member of (26) is an upper bound of the round-off errors for both the explicit analog (5) and the implicit analog (6).

In the case of the explicit scheme (5) the matrix $M$ of (13) is the matrix $A$ appropriate to (5) while the matrix $N$ of (13) is $=-I$ where $I$ is the $M \times M$ identity matrix. The eigenvalues of $A$ are known [2] to be

$$
\lambda_{r}=2-4 R^{2} \cos \frac{r \pi}{2(M+1)}
$$

The eigenvalues of $-I$ are clearly $=-1$. Thus the quadratic equation (18) becomes

$$
x^{2}-\left[2-4 R^{2} \cos \frac{r \pi}{2(M+1)}\right] x+1=0 .
$$

It is clear that if the roots of (28) were real, one would have to be larger than unity, since the products of the roots is $=1$. Under these conditions $\beta_{r}(p)$ as defined in (22) would not be bounded as $p \rightarrow \infty$ and the difference scheme (5) could not be stable. Thus the roots of (28) must be complex, in which case the modulus of the roots is $=1$ and $\beta_{r}(p) \leqq p+1$.

An upper bound of the round-off errors after $n$ time steps is then given by

$$
E_{n}^{*}=\frac{1}{2}(n+1)(n+2) \sqrt{M} \delta^{*}
$$

where $\delta^{*}=3 \times \frac{1}{2} \times 10^{-p}$ if the computations are carried to $p$ decimal places. In the case of the implicit scheme (6) matrices $M$ and $N$ of (13) are $A^{-1}$ and $A^{-1} B$ respectively where the matrices $A$ and $B$ appropriate to (6) have been defined earlier.

It can be easily shown that the matrices $A^{-1}$ and $A^{-1} B$ have the same eigenvectors, as required in the above developments $[2$, p. 20], and that their eigenvalues are

$$
\lambda_{r}=2 /\left(1+2 R^{2} \cos ^{2} \frac{r \pi}{2(M+1)}\right) ; \quad \mu_{r}=-1
$$

Thus the quadratic equation (18) becomes

$$
x^{2}-\frac{2}{1+2 R^{2} \cos ^{2} \frac{r \pi}{2(M+1)}} x+1=0 .
$$

Clearly the roots of (30) must be complex. This leads to the condition

$$
1 /\left\{1+2 R^{2} \cos [r \pi / 2(M+1)]\right\}<1
$$

which is evidently satisfied for any value of $R$. Thus the difference scheme (6) is unconditionally stable. Furthermore, and for the same reason as above,

$$
\beta_{r}(p) \leqq p+1
$$

An upper bound of the round-off errors after $n$ time steps is, therefore, once more 
given by

$$
E^{*} \leqq \frac{1}{2}(n+1)(n+2) \sqrt{M} \delta^{*} .
$$

In this case, however, as previously mentioned $\delta^{*}$ is an upper bound of the errors which arise both from the use of approximations to $A^{-1}$ and $A^{-1} B$ in lieu of exact matrices and from the process of rounding-off the values of the products and quotients involved in the evaluation of the components of $\mathbf{u}_{k+1}$. Clearly $\delta^{*}$ depends on the specific scheme for solving the system of equations (6) with $h=1,2,3, \cdots M$ for the $u_{h, k+1}$ 's.

In order to estimate $\delta^{*}$ for the implicit scheme we note that the counterpart of the typical equation (9) is

$$
\mathbf{u}_{k}^{*}=4 A^{-1} \mathbf{u}_{k-1}^{*}+A^{-1} B \mathbf{u}_{k-2}^{*}+\boldsymbol{\delta}_{k}
$$

whence

$$
A \mathbf{u}_{k}^{*}=4 \mathbf{u}_{k-1}^{*}+B \mathbf{u}_{k-2}^{*}+A \boldsymbol{\delta}_{k} .
$$

Let $\mathbf{R}_{k}$ denote the known vectors $A \mathbf{u}_{k}{ }^{*}-4 \mathbf{u}_{k-1}^{*}-B \mathbf{u}_{k-2}^{*}$. Then $A \boldsymbol{\delta}_{k}=\mathbf{R}_{k}$ and therefore $\boldsymbol{\delta}_{k}=A^{-1} \mathbf{R}_{k}$. Since the eigenvalues of $A$ are known to be larger than 2 , it follows that the eigenvalues of $A^{-1}$ are smaller than unity and therefore

$$
\left\|\boldsymbol{\delta}_{k}\right\|=\left\|A^{-1} \mathbf{R}_{k}\right\| \leqq\left\|\mathbf{R}_{k}\right\| \text {. }
$$

We conclude that $\delta^{*}$ in equation $\left(26^{*}\right)$ is the largest of the norms of the $n$ "residual vectors" $\mathbf{R}_{k}=A \mathbf{u}_{k}{ }^{*}-4 \mathbf{u}_{k-1}^{*}-B \mathbf{u}_{k-2}^{*}$. These vectors will depend, of course, on the specific method of computing the $\mathfrak{u}_{k+1}$ 's from (6).

A discussion of two alternative schemes for solving implicit systems of equations of the type (6) is contained in [3].

Yeshiva University

New York, New York; and

AVCO Corporation

Wilmington, Massachusetts

1. R. D. Richtmeyer, Difference Methods for Initial-Value Problems, Interscience Publishers, Inc., New York, 1957.

2. A. N. Low AN, The Operator Approach to Problems of Stability and Convergence, Scripta Mathematica, Yeshiva University, New York, 1957, p. 55, p. 82-86.

3 . A. N. Low AN, "On the propagation of round-off errors in the numerical integration of the heat equation," Math. Comp., v. 14, 1960, p. 139-146. 